\title{
Proceedings of the 151st Semon Club, 23rd May 2016, ENT Department, Guy's and St Thomas' NHS Foundation Trust, London, UK
}

\author{
Chairperson: Mr Elfy B Chevretton, Guy's and St Thomas' NHS Foundation Trust \\ Secretary: Dr Sherif Haikel, Royal National Throat, Nose and Ear Hospital, University College London Hospitals NHS Trust \\ Invited panel for pathology: Dr Ann Sandison, Charing Cross Hospital, Imperial College Healthcare NHS Trust \\ Invited panel for radiology: Dr Steve Connor, and Dr Ata Siddiqui, Guy's and St Thomas' NHS Foundation Trust \\ Professor Leslie Michaels prize for the best presentation of the meeting was awarded to Andrew Hoey for 'Progressive aggressive labyrinthitis'.
}

The chairperson and secretary have edited the proceedings of the meeting to reflect the discussion of each case by the expert panel and audience during the Semon Club meeting.

Head and neck section

Chairperson: Mr Richard Oakley

Recurrent upper respiratory tract infections after complex surgery for recurrent metastatic oesophageal carcinoma

S Clark, H Jones, K Ghufoor

From the Royal London Hospital

\section{Introduction}

We present the diagnostic challenge of a patient who developed recurrent chest infections with an unusual aetiology months after surgery for recurrent oesophageal carcinoma. This case is particularly interesting as we believe the specific complication has not been previously reported.

\section{Case report}

A 57-year-old gentleman underwent an oesophagectomy with gastric pull-up for a tumour-node-metastasis stage $\mathrm{T}_{3} \mathrm{~N}_{1} \mathrm{M}_{0}$ lower oesophageal adenocarcinoma. Two years later, he developed an extensive recurrence that was treated surgically with laryngo-pharyngo-oesophagectomy and colonic interposition. Ten months later, he developed recurrent chest infections and sputum production from the stoma. Initial tracheoscopy confirmed lower respiratory tract infection, but showed no fistulation. He was primarily treated with antibiotics, but this did not fully resolve his symptoms.

\section{Radiological findings}

A contrast swallow study confirmed there was no fistulation between the respiratory tract and the colonic interposition. However, subsequent contrast computed tomography demonstrated a fistulous connection between the trachea and an air/fluid-filled viscus not visible on the swallow study. This was later confirmed to be a fistula originating from the gastric remnant that remained in the thorax after the initial gastric pull-up.

\section{Histological findings}

The recurrent oesophageal tumour was demonstrated to be a poorly differentiated, highly infiltrative adenocarcinoma.

\section{Management}

The fistula was closed successfully via endoscopic tracheoscopy using collagen injections and fibrin sealant. Unfortunately, the patient developed a large burden of metastatic disease and therefore the gastric remnant was not resected.

\section{Discussion and conclusion}

Professor Howard pointed out the importance of understanding the natural history of a disease. Lower oesophageal adenocarcinoma is known to spread to the stomach. A fistula from the gastric remnant in the thorax would therefore be more likely than a fistula from the colon. Mr Oakley said there is currently debate among head and neck surgeons as to the suitability of surgical palliation for cases like this.

\section{An unusual case of stridor}

S Shah, G H Jones, J Tahery

From the Countess of Chester Hospital NHS Foundation Trust

Introduction

Stridor is an ENT emergency that requires prompt assessment and management. We present a case of occult stridor in a challenging adult patient.

\section{Case report}

A 40-year-old female presented to the clinic with a 6-month history of shortness of breath and a 2-week history of quiet inspiratory stridor. Her background included asthma, learning difficulties and health anxiety disorder. The findings of ENT examinations, including flexible nasendoscopy, were unremarkable. A magnetic resonance imaging (MRI) scan demonstrated a left thyroid nodule causing compression of the trachea to $6 \mathrm{~mm}$. The patient subsequently underwent urgent hemi-thyroidectomy. Six hours post-operatively, she re-developed stridor and was admitted to the intensive care unit. Extubation failure prompted a surgical tracheostomy and direct laryngoscopy. A soft-tissue mass was visualised in the subglottic region originating from the posterior tracheal wall. This mass was biopsied. 


\section{Radiological findings}

The pre-operative MRI scan was reported as showing a left thyroid nodule between the trachea and oesophagus causing tracheal narrowing. The persistence of the lesion associated with the posterior wall of the trachea was, however, still present on the post-operative computed tomography scan. Dr Connor felt that a separate nodule was present between the oesophagus and the trachea, and this was responsible for the tracheal compression, as it is unusual for thyroid nodules to behave in this way.

\section{Histological findings}

Initial histology from the intraluminal lesion suggested a capillary haemangioma, but a second opinion indicated that it was traumatic in origin.

\section{Management}

The patient was referred to a laryngologist with an interest in tracheal surgery. The patient is awaiting excisional biopsy.

\section{Discussion and conclusion}

Mr Haikel advised the pre-operative visualisation of the trachea in all cases of stridor with a thyroid lesion. Professor Howard felt that the patient could have been treated with an emergency tracheostomy, if necessary, at presentation, and then awaited multidisciplinary team discussion for further management. He repeated the importance of inserting a 0 -degree Hopkins rod past the glottis, and would have suggested the biopsy of this airway mass during the tracheoscopy. He said that in adults, most primary tracheal tumours are malignant, and adenoid cystic carcinoma is the most common type.

\section{Slow-growing asymptomatic neck lump}

\section{R C T Cheong, P Bowles, A Pelser}

From the Brighton and Sussex University Hospitals NHS Trust, Brighton

\section{Introduction}

Schwannoma arising from the cervical sympathetic chain is uncommon; approximately 57 cases have been reported. The term 'ancient' schwannoma was introduced by Ackerman and Taylor in their description of 10 benign tumours demonstrating extensive hyalinisation suggestive of degenerative changes.

\section{Case report}

A 36-year-old female presented to the ENT 2-week wait clinic with a 1-month history of a lump on the left side of her neck. There were no other associated symptoms or significant history.

\section{Radiological findings}

A magnetic resonance imaging (MRI) scan showed a $4.8 \times$ $1.8 \times 2.1 \mathrm{~cm}$ heterogeneous soft-tissue mass arising in the region of level II, with enhancement post contrast. Dr Connor said that sympathetic schwannomas usually push the carotid artery anteriorly or laterally, and that the location was unusual for a schwannoma.

\section{Management}

At surgery, careful dissection was made down onto the mass. The vagal nerve was identified, and the mass was found to be arising from the cervical sympathetic chain. The mass was peeled away from the nerve sheath, whilst attempting to cause minimal trauma to the nerve tissue.

\section{Histological findings}

Ancient schwannomas are schwannomas that show pronounced degenerative change. Dr Sandison said that needle aspiration of a sympathetic schwannoma is often very painful. The degenerative changes can cause nuclear atypia that can be misdiagnosed as malignant. For these reasons, she would advise excisional biopsy of the whole lesion whenever possible.

\section{Discussion}

Dr Sandison commented that the clinical management of the different histological subtypes of schwannomas would not differ significantly. Mr Oakley held the opinion that it was important to balance the small risk of potential malignancy against that of post-operative Horner's syndrome. He also advised a screening MRI in any young patients with a schwannoma, to exclude neurofibromatosis type 2 .

Salbutamol does not work for primary tumours of the cervical trachea

R Lakhani, K Smith, R Simo

From the Guy's and St Thomas' NHS Foundation Trust, London

\section{Introduction}

Primary tracheal tumours are rare. Tracheal lesions are more commonly encountered secondary to infiltration from adjacent structures such as the thyroid gland.

\section{Case report}

A 42-year-old lady presented with a 6-month history of difficulty in breathing and decreased exercise tolerance. Her symptoms worsened despite treatment for suspected asthma. Her swallow was fine, but she had lost weight over the six months. Examination revealed biphasic stridor, an otherwise normal upper aerodigestive tract and no cervical lymphadenopathy. Urgent imaging was organised for the same day in light of the clinical presentation.

\section{Radiological findings}

Computed tomography and magnetic resonance imaging scans were obtained. A well-defined exophytic lesion was seen within the upper trachea (thoracic vertebrae T1-2 level), located approximately $2.2 \mathrm{~cm}$ below the cricoid and $4 \mathrm{~cm}$ above the carina. It was significantly narrowing the tracheal lumen. The lesion measured $2.4 \times 1.7 \times 1.5 \mathrm{~cm}$. The lesion did not extend beyond the wall of the trachea. There were no enlarged cervical lymph nodes. The adjacent thyroid gland showed some non-specific nodules bilaterally.

\section{Histological findings}

The patient underwent a tracheoscopy and bronchoscopy, and excisional biopsies were performed. Her tracheal lumen was found to be 90 per cent occluded. Biopsies revealed adenosquamous carcinoma of the trachea (tumournode-metastasis stage $\mathrm{T}_{2} \mathrm{~N}_{0} \mathrm{M}_{0}$ ). Dr Sanderson demonstrated the adenosquamous morphology of this high-grade epithelial tumour, and explained how complete resection is difficult because of the likelihood of extensive spread. These tumours are poorly radiosensitive. Professor Howard commented that approximately 50 per cent of primary tracheal tumours are squamous and 50 per cent are adenoid cystic. 


\section{Management}

The patient underwent a segmental tracheal resection, level VI neck dissection and primary end-to-end anastomosis of the cervical trachea.

\section{Discussion and conclusion}

Mr Oakley suggested consideration of more aggressive surgery, with an additional thyroidectomy. Professor Howard felt the prognosis was so poor that he advised against more aggressive surgery; he felt quality-of-life issues were more important.

\section{A rare, incidental finding of a sinonasal mass}

\section{$\mathrm{N}$ Caton, B Fu}

From the East Kent Hospitals University Foundation NHS Trust, Canterbury

\section{Introduction}

Peripheral nerve sheath tumours of sinonasal mucous membranes are rare neoplasms. They can be asymptomatic, or present with nasal obstruction, epistaxis or anosmia.

\section{Case report}

Magnetic resonance imaging performed to investigate dizziness in an 80-year-old gentleman revealed an incidental finding of an ethmoid sinus mass. Examination indicated a polypoidal lesion lateral to the middle turbinate.

\section{Radiological findings}

A computed tomography scan of the sinuses revealed a nodular, ovoid, soft-tissue opacity in the left anterior ethmoidal sinus, with bowing of the adjacent bony structures. Dr Connor said that bony remodelling signifies a benign or low-grade malignant lesion.

\section{Management}

The patient underwent left endoscopic polypectomy for histology. The patient is currently under clinical surveillance to assess for recurrence.

\section{Histological findings}

The nasal polyp was composed of atypical spindle cells, with nuclear pleomorphism and a fascicular growth pattern. Immunohistochemistry showed patchy positive staining for S-100 protein, which indicates a neural tumour. The findings are consistent with a malignant peripheral nerve sheath tumour, but the lack of mitoses was unusual. However, the marked pleomorphism did suggest the possibility of a lowgrade malignant peripheral nerve sheath tumour. Professor Cyril Fisher (Royal Marsden Hospital, London) agreed with this diagnosis of exclusion.

\section{Lessons learnt}

Low-grade malignant nerve sheath tumours are rare causes of nasal lesions. Endoscopic endonasal surgical resection is feasible if contained within the sinonasal cavity. If there is evidence of intradural or intracranial extension, more extensive surgery via an endonasal transcribriform and transpterygoid or lateral rhinotomy approach is required.

Cranial nerve palsies, maxillary antrum mass and thoracic bony lesions: a head and neck conundrum

N Singh, N Orban, A Sandison

From the Charing Cross Hospital, London

\section{Introduction}

Rhabdomyosarcoma is rare, usually affects children, and shows predilection for the head and neck. Presenting symptoms are site-dependent, and may mimic other benign and malignant conditions. Survival is dependent on early diagnosis and aggressive multimodality treatment.

\section{Case report}

A normally well 44-year-old man presented with suddenonset right hypoglossal nerve palsy, an altered sensation over the maxillary distribution of the trigeminal nerve and a firm $2 \mathrm{~cm}$ level II neck node. He described a two-month history of mid-thoracic back pain and $10 \mathrm{~kg}$ weight loss. Blood analysis showed raised inflammatory markers and marked hypercalcaemia.

\section{Radiological findings}

A magnetic resonance imaging scan demonstrated a tumour in the right nasal cavity and maxillary sinus, extending to the right foramen rotundum and right infratemporal fossa. There was bone involvement at the left hypoglossal canal, and multiple lytic lesions in the cervical and lumbar vertebrae, with collapse of the ninth thoracic vertebra.

\section{Histological findings}

Biopsy from the right maxillary antrum showed high-grade malignant pleomorphic tumour cells that stained positive for desmin skeletal muscle markers, consistent with a diagnosis of rhabdomyosarcoma, possibly alveolar subtype.

\section{Management}

This patient was initially treated symptomatically with antibiotics, intravenous fluids and bisphosphonate therapy to correct the hypercalcaemia. He was referred urgently to the local sarcoma unit. After multidisciplinary team discussion, he was counselled initially for palliative radiotherapy, with a view to progressing to palliative chemotherapy.

\section{Discussion}

Dr Sandison commented that rhabdomyosarcoma is unusual for a sarcoma in that it does metastasise to the lymph nodes. Prof Howard advised that one should also be wary of missing the diagnosis of rhabdomyosarcoma in children who present with periorbital cellulitis to the on-call ENT team. He reminded the audience that ENT surgeons see more children than paediatric surgeons, and so must be aware of this condition. Indeed, most cases of rhabdomyosarcoma are diagnosed by ENT surgeons.

\section{Rhinology and anterior skull base section}

Chairperson: Mr Shahzada Ahmed

\section{A rare, benign cause of unilateral nasal obstruction}

$\mathrm{N}$ Caton, B Fu

From the East Kent Hospitals University Foundation NHS Trust, Canterbury

\section{Introduction}

Nasal chondromesenchymal hamartoma is a rare cause of benign sinonasal lesions. It typically presents in infants, but we present a case in an adult. Patients tend to present with symptoms related to compression of the nasal chondromesenchymal hamartoma on local structures within the nasal cavity and paranasal sinuses. 
Case report

A 47-year-old gentleman presented to the clinic with several months history of left nasal obstruction, clear rhinorrhoea and reduced sense of smell. Flexible nasendoscopy revealed a large left-sided nasal polyp, which appeared benign with a smooth mucosa. The septum was deviated to the right due to the mass effect of the polyp.

\section{Radiological findings}

A computed tomography scan demonstrated soft-tissue opacity with inflammatory changes within the left nasal cavity, which appeared to arise from the nasal septum and extend into the inferior meatus and nostril. Dr Connor demonstrated some moulding of bone, but there was no bony erosion. Dr Connor also advised that any nasal lesion that extends to the skull base, as this did, should be evaluated with magnetic resonance imaging to exclude a meningocele.

\section{Management}

The patient underwent an urgent left endoscopic polypectomy for histology. The polyp was completed excised. Subsequent clinic surveillance has not demonstrated any recurrence to date.

\section{Histological findings}

The nasal polyp biopsy showed irregular lobulated proliferation of hyaline and fibrous cartilage set in a myxoid and partly fibrous stroma. These features suggest nasal chondromesenchymal hamartoma. However, the possibility of a cartilaginous neoplasm could not be entirely ruled out. A specialist opinion was requested and a diagnosis of nasal chondromesenchymal hamartoma was confirmed. Dr Sanderson pointed out the intact mucosa, the lack of necrosis or mitosis, and the bland cells as signs of a benign lesion.

\section{Conclusion}

Nasal chondromesenchymal hamartomas can mimic other benign or malignant lesions within the sinonasal cavity. Surgical resection is the preferred management and the recurrence rates are very low. Professor Howard reminded the audience of the importance of sending all nasal polyps for histology. Mucus-secreting adenocarcinoma looks grossly like an allergic polyp.

\section{Epiphora of nasal origin}

A Pora, M Alfikey, P Prinsley

From the Norfolk and Norwich University Hospital, Norwich

\section{Case report}

A 76-year-old male patient presented to the ophthalmology clinic with a 3-month history of right-sided epiphora secondary to chronic dacryocystitis. He was scheduled for endoscopic dacryocystorhinostomy. The nasal cavity was not inspected at this stage. Nasal endoscopy in the operating theatre revealed a large polypoid mass, arising from the lateral surface of the inferior turbinate, which obstructed the opening of the nasolacrimal duct at the inferior meatus. Dacryocystorhinostomy was not performed. All visible neoplasm was resected and a Monoka stent was inserted into the nasolacrimal duct.

\section{Radiological findings}

A post-operative computed tomography (CT) scan of the orbit with contrast showed dilation of the right nasolacrimal canal compared to the left, with dacryocystocele formation and soft-tissue thickening, consistent with chronic nasolacrimal duct obstruction.

\section{Histological findings}

Dr Sandison explained that oncocytes arise from the epithelial cells of salivary ducts. Minor salivary glands exist around the opening of the nasolacrimal duct. There were no features of malignancy. These findings are consistent with a nasal oncocytoma. This tumour type is typically confined to the salivary glands and kidneys, with only 17 examples reported as originating within the nose.

\section{Management}

Following removal of the oncocytoma, this gentleman's epiphora improved significantly. This case highlights the potential need for all patients presenting with epiphora to have an ENT review before management in order to rule out nasal causes of nasolacrimal duct obstruction. This would enable pre-operative imaging and more accurate diagnosis, guiding management if nasal pathology was detected.

\section{Discussion}

Following discussion at the Semon Club, it was suggested that rather than direct ENT review, it may be more appropriate to arrange CT imaging of the nasolacrimal duct for all epiphora patients prior to deciding on management.

\section{Riedel's procedure - the importance of cosmesis in facial surgery}

A Nassimizadeh, M Nassimizadeh, S K Ahmed

From the University Hospitals Birmingham NHS Foundation Trust

\section{Introduction}

Although sinus surgery was first introduced by the ancient Egyptians, the first modern description of frontal sinus surgery can only be traced to 1750 . In 1898 , Riedel advocated complete removal of the anterior table and floor of the frontal sinus, whilst simultaneously stripping the mucosa. The major post-operative problem was with gross forehead deformity, and this limited its use. With advances in endoscopic sinus surgery, the need for open procedures diminished further. We describe a modification of Riedel's procedure that gives much-improved cosmetic outcomes.

\section{Case report}

A 74-year-old male had previously undergone frontal sinus surgery for chronic rhinosinusitis and a left frontal mucocele. $\mathrm{He}$ re-presented with recurrent sinusitis and a left frontoorbital fistula draining into the supra-medial orbit. Because of the small anteroposterior distance of the frontal sinus and the extensive neo-osteogenesis secondary to chronic frontal sinusitis, an endonasal endoscopic approach was not possible. Following multidisciplinary discussion, the patient underwent Riedel's procedure with excision of the fronto-orbital fistula. In order to reduce the post-operative cosmetic defect, a thick vascularised pedicle graft, which included periosteum and galea muscle, was used to obliterate the dead space after removal of the anterior frontal sinus wall.

\section{Radiological findings}

A computed tomography scan of the sinuses showed a small anteroposterior distance of the frontal sinus and extensive neo-osteogenesis secondary to chronic frontal sinusitis. 


\section{Conclusion}

We believe the surgical modification to Riedel's classical procedure, as described above, results in improved cosmetic outcomes. It should remain in the armamentarium of rhinologists treating complex frontal sinus disease.

\section{A case of 'special' right nostril polyp}

D Yap, S Farmer

From the Royal Gwent Hospital, Newport, Wales

\section{Introduction}

Seromucinous hamartoma is a rare, benign, glandular proliferation of the sinonasal tract and, rarely, nasopharynx, arising from submucosally situated seromucinous glands. At present, there are 23 reported cases in the English literature and less than 25 identified in the world literature. We present an additional case report of seromucinous hamartoma.

\section{Case report}

A 34-year-old man presented with a 2-year history of bilateral nasal obstruction. He had associated anosmia, with no evidence of rhinorrhoea, epistaxis or pain. He was a nonsmoker, fit and healthy, with no known allergies. On examination, there was reduced airflow into the right nostril. Flexible nasendoscopy revealed abnormal soft tissue in the right nasopharynx. Examination under anaesthesia showed polypoidal tissue filling the post-nasal space arising from a right accessory maxillary sinus ostium.

\section{Radiological findings}

Radiology revealed mucosal thickening of the floor of the right maxillary antrum, with soft tissue extending into the nasal cavity and inferior turbinate. There were also several polyps arising from the superior and middle meati.

\section{Histological findings}

Histology revealed a bosselated polypoid lesion lined with mature pseudostratified columnar epithelium, with hyalinisation along the basement membrane. The connective tissue was predominantly fibrous, with focal fibromyxoid tissue and mature seromucinous glands. Dr Sandison explained that a hamartoma is a proliferation of normal structures.

\section{Conclusion}

Any nasal polyp should be sent for histological diagnosis. The patient will be followed up with a repeat computed tomography scan after two years, and will be discharged if no recurrence is detected.

\section{Progressive nasal destruction}

U Qazi, D Walker, L Pitkin

From the Royal Surrey County Hospital, Guildford

\section{Introduction}

We present a case of nasal natural killer/T cell lymphoma presenting with a short history.

\section{Case report}

A 95-year-old lady was initially referred to the clinic with hearing loss. In the interim period, she developed a painless, nasal swelling. The swelling grew rapidly, causing progressive nasal obstruction, rendering nasoendoscopy impossible.
Within two weeks of presentation, she complained of blurred vision, indicating orbital involvement.

\section{Radiological findings}

A computed tomography (CT) scan of the sinuses revealed a large soft-tissue lesion arising from the right nasal ala, with differential diagnoses including neoplasia, inflammation and infection. Nasal lymphoma was considered to be more likely than squamous cell carcinoma because of the lack of bony destruction. Two weeks later, a repeat CT scan showed that the lesion had increased in size, and was now infiltrating the medial right orbit.

\section{Histological findings}

Initial biopsies proved indeterminate as the tissue was necrotic. Subsequent specimens showed diffuse infiltration by pleomorphic lymphoid cells, with large areas of necrosis. Cells had a high rate of proliferation and were positive for Epstein-Barr virus. The overall appearance was of a highgrade extranodal natural killer/T cell lymphoma.

\section{Management}

Disease extent and its rapid progression deemed chemoradiotherapy treatment unsuitable. The patient died a week later.

\section{Discussion and conclusion}

Professor Howard commented that non-specific early symptoms and radiology findings make early diagnosis challenging. Nevertheless, if the diagnosis of $\mathrm{T}$ cell lymphoma is confirmed and treated in a timely manner, the disease should not be considered fatal. The now obsolete term for this disease, 'lethal midline granuloma', may confer the aggressive nature of the disease. However, it is also misleading as the lesions are not always lethal, or in the midline, and they are not granulomas.

A rare cause for maxillary pain, nasal obstruction and deteriorating visual acuity

K Lee, N Mani, N Slevin

From the Christie Hospital, Manchester

\section{Introduction}

Amyloidosis is a group of heterogeneous diseases resulting from the accumulation of misfolded proteins. Although rare, 20 per cent of cases involve head and neck structures. Currently, treatment of local amyloidosis focuses around surgical resection; however, optimum treatment is unknown for cases that are not amenable to surgery.

Case report

A 40-year-old lady presented with a 2-year history of gradual maxillary pain and nasal obstruction, with a recent reduction in visual acuity.

\section{Radiological findings}

Magnetic resonance imaging showed a lesion in the left pterygopalatine fossa, extending into the orbital apex and cavernous sinus.

\section{Histological findings}

Biopsy demonstrated green birefringence when viewed under polarised light after Congo red dye staining. 


\section{Management}

The diagnosis was localised left pterygopalatine amyloidosis. Given the progressive eye symptoms in this case, conservative management was not an option, and excision would have resulted in significant morbidity for benign disease. A trial of radiotherapy was therefore started based on reports of successful use in other head and neck cases. This resulted in involution of the amyloidosis and marked improvement of symptoms. Follow-up imaging at five years demonstrated no evidence of progression.

\section{Discussion}

Mr Ahmed felt surgery could have been possible; however, given the successful results following radiotherapy, he felt this did provide a suitable alternative treatment option. He also suggested joint management of such cases with a rheumatologist.

\section{Conclusion}

This is the first documented case of pterygopalatine fossa amyloidosis successfully treated with radiotherapy. Following multidisciplinary discussion, radiotherapy should be considered as an alternative treatment option for localised amyloidosis.

\section{Otology and lateral skull base section}

Chairperson: Miss Irumme Pai

\section{A cerebellopontine angle conundrum}

H R Lancer, H R F Powell, S R Saeed

From the Royal National Throat, Nose and Ear Hospital, London

\section{Introduction}

Cerebellopontine angle tumours are the most common neoplasms of the posterior fossa. They account for 5-10 per cent of intracranial tumours, and over 90 per cent are vestibular schwannomas or meningiomas.

\section{Case report}

A 70-year-old female with a past medical history of melanoma presented in August 2014 with episodic vertigo, nausea and hearing loss, with progression 3 months later to transient left-sided facial weakness and nystagmus. Crosssectional imaging was arranged. In January 2015, the skull base multidisciplinary team (MDT) suggested this was likely to be a case of metastasis or inflammatory polyneuropathy. Further imaging and a lumbar puncture were advised. In February 2015, there was recurrence of facial palsy, and progressive left-sided sensorineural hearing loss, with the onset of diplopia and ophthalmoplegia on left lateral gaze. This led to expedient admission under neurology; there had been multiple non-diagnostic lumbar punctures. In April 2015, the skull base MDT advised biopsy via a retrolabyrinthine route.

\section{Radiological findings}

Magnetic resonance imaging (MRI) conducted in December 2014 revealed a fusiform enhancing tumour, extending from the left cerebellopontine angle along the internal auditory meatus, and involving the cochlea, vestibule and semicircular canals. In January 2015, MRI indicated regression of the cerebellopontine angle lesion - a possible inflammatory process. An MRI performed in April 2015 revealed progression of the enhancing cerebellopontine angle lesion, featuring surrounding oedema and thickening of the left VIIth,
VIIIth and Vth cranial nerves, with extension into Meckel's cave.

\section{Histological findings}

Histology conducted in April 2015 revealed a diffuse large B cell lymphoma.

\section{Management}

The patient was referred to the lymphoma team for chemotherapy.

\section{Conclusion}

Obtaining tissue for a histological diagnosis is warranted for some cerebellopontine angle lesions. A retrolabyrinthine (otic capsule sparing) approach is possible with favourable petrous temporal anatomy. Specialist review of imaging and discussion in a skull base MDT are essential when dealing with unusual cerebellopontine angle lesions. Miss Pai enquired if steroid treatment can affect the histology of lymphoma. Dr Sandison confirmed that steroids can cause necrosis of the most aggressive parts of a tumour and can also influence the immune profiling panel.

\section{An aggressive recurrent cholesteatoma?}

H R Lancer, N Amin, I Pai

From the Guy's and St Thomas' NHS Foundation Trust, London

\section{Introduction}

Cholesteatoma is a cystic, inflammatory mass of keratinising stratified squamous epithelium, which, if untreated, can cause significant erosion of the surrounding structures.

\section{Case report}

A 79-year-old male presented with a history of advanced cholesteatoma that required a right modified radical mastoidectomy in September 2015. Despite macroscopic clearance of disease, the patient had ongoing symptoms and was scheduled for a revision procedure three months later. In February 2016, whilst on the waiting list, he was seen in the out-patient clinic with a new lower motor neuron facial nerve palsy and intractable otalgia, on a background of longstanding, progressive balance disturbance. The patient underwent a right subtotal petrosectomy 9 days later, which revealed a large volume of keratin extending into the internal auditory meatus. There was significant bony destruction, and adhesions to the dura, internal carotid artery, and tympanic and mastoid segments of the facial nerve. A subsequent petrosectomy with blind sac closure was performed.

\section{Radiological findings}

Dr Connor demonstrated a computed tomography (CT) scan from September 2015 consistent with cholesteatoma, although he did note some bony erosion of the superior semicircular canal that is suspicious. A CT scan conducted in February 2016 showed an extensive erosive process involving the right temporal bone and posterolateral skull base, extending to the temporomandibular joint and surrounding soft tissues. A magnetic resonance imaging scan showed intracranial dural involvement, and thrombosis of the jugular bulb and sigmoid sinus was noted.

\section{Histological findings}

The histological findings from September 2015 indicated cholesteatoma. The findings from February 2016 revealed well-differentiated squamous cell carcinoma (SCC). Dr Sandison demonstrated how some of the features of the 
well-differentiated SCC were visible, although less pronounced, in the pathology from the first specimen in 2015, and commented on how these features can resemble an inflammatory process.

\section{Management}

The head and neck multidisciplinary team advised palliative radiotherapy.

\section{Conclusion}

In such rapid and aggressive disease, a malignant process should be considered as a differential diagnosis; hence, sufficient tissue specimens must be sent for histopathological evaluation. Dr Sandison explained that SCCs originating in the external auditory canal can grow around the tympanic membrane and into the temporal bone.

\section{An expanding posterior auricular lump}

M Monem, B Mettias, M Lyons

From the Lister Hospital, Stevenage

\section{Introduction}

We discuss the management of a posterior auricular lump, of which there have been just over 130 documented cases.

\section{Case report}

A 22-year-old Caucasian female engineer presented with an expanding lump in the left post-auricular area, which had been uncomfortable for 6 months and interfered with the wearing of sunglasses. There were no hearing, balance, meningitic or constitutional symptoms reported. There was a past medical history of anorexia. The patient was a non-smoker and there was no family history of cancers. On examination, there was a 3-4 cm, well-demarcated, firm-to-hard, nonmobile tumour in the left post-auricular region. There were no signs of inflammation and no lymphadenopathy. Cranial nerve function was normal. Examination findings of the oropharynx, nasopharynx and laryngopharynx were unremarkable.

\section{Radiological findings}

A computed tomography scan of both mastoids demonstrated a well-circumscribed, dense, bony, sessile growth originating from the outer cortex of the left mastoid process, with a wide base or stalk. A diffusion-weighted magnetic resonance imaging scan confirmed this.

\section{Histological findings}

The macroscopic appearance was consistent with bony tissue. Microscopy showed sections of bone including Haversian canals. There were no features of malignancy.

\section{Management}

A diagnosis of a mastoid osteoma was made. This was managed with excision of the tumour. A good functional outcome has been achieved, with no recurrence. This management is in line with current practice.

Now you see the drum, now you don't. A case of rapidonset conductive hearing loss in a child

N C Seymour, R Tranter, K Argiris

From the Brighton and Sussex University Hospitals NHS Trust, Brighton

\section{Introduction}

Fibrous dysplasia is a benign disease of the bones, which affects the differentiation and maturation of osteoblasts. Fibrous dysplasia can affect the craniofacial bones, which can be asymptomatic or present with symptoms commonly found in an ENT out-patient clinic.

\section{Case report}

A 10-year-old presented with right-sided hearing loss of rapid onset. On examination, there was a narrowing of the external auditory canal that had visibly narrowed within one month. An audiogram showed moderate conductive hearing loss.

\section{Radiological findings}

High-resolution computed tomography showed a stenosis of the right external auditory canal secondary to expansion, and ground-glass opacification of the petrous temporal bone. Dr Siddiqui demonstrated the features of fibrous dysplasia: the ground-glass expansion of the temporal bone, with an intact cortex that spares the otic capsule, and a lack of periosteal reaction.

\section{Management}

Surgery for craniofacial fibrous dysplasia is reserved for patients with significant hearing loss, complications or deformity.

\section{Discussion}

Other cases have been reported of temporal bone fibrous dysplasia, but our case is unique in its presentation. Dr Siddiqui said he would advise against biopsy of these lesions. Miss Pai advised against surgery whenever possible, as these lesions tend to regrow. Miss Chevretton advised that if surgery is necessary in this patient, it is best to wait until after puberty.

\section{Conclusion}

Fibrous dysplasia of the temporal bone is uncommon. Many cases in other series were found incidentally from radiology reports; therefore, it is a diagnosis that may be overlooked.

A rare presentation of sudden, bilateral sensorineural hearing loss, hemi-facial palsy and vertigo

J Godbehere, T Kanzara, B F Jones

From the Arrowe Park Hospital, Birkenhead

\section{Introduction}

Sudden sensorineural hearing loss is a common presentation to ENT departments. Bilateral involvement and associated cranial nerve deficits are rare, and could suggest complex pathology.

\section{Case report}

A 54-year-old Caucasian, fit and well female presented with progressive, bilateral hearing loss, tinnitus and left-sided facial palsy, with gradual onset of rotatory vertigo over 3 months. She demonstrated gross impairment of her balance and a grade III House-Brackmann left-sided facial palsy. Pure tone audiogram confirmed a dead right ear and severe left-sided sensorineural hearing loss.

\section{Radiological findings}

A magnetic resonance imaging scan showed: meningeal enhancement in both internal auditory canals, enhancement of the geniculate and tympanic portions of the left facial 
nerve, and enhancement of the right trigeminal nerve within Meckel's cave.

\section{Histological findings}

Syphilis serology was positive.

\section{Management}

After initial failure to respond to oral steroids prior to positive syphilis serology results, treatment with penicillin $\mathrm{V}$ was commenced. Hearing in the left ear was aided with an air conduction hearing aid.

\section{Discussion}

Syphilis is not always screened for in sudden sensorineural hearing loss, despite increasing incidence in the UK. Because of a latent period between primary infection and neurosyphilis, symptoms of a genitourinary infection may not be elicited. Early detection may prevent potentially irreversible hearing loss.

\section{Conclusion}

Given the increased incidence and latency in presentation, it is important to screen for syphilis in patients with sudden sensorineural hearing loss, even where no history of a genitourinary infection is elicited.

\section{Progressive aggressive labyrinthitis}

A W Hoey, C Xie, I Pai

From the Guy's and St Thomas' NHS Foundation Trust, London

\section{Introduction}

Balance disturbance, facial nerve palsy and profound hearing loss are rare but well-recognised complications of mastoid surgery. If they do occur, they are usually apparent in the immediate post-operative period.

\section{Case report}

A 55-year-old female presented with a long history of recurrent ear infections. She was found to have an attic cholesteatoma and mild conductive hearing loss. The patient underwent an uneventful combined approach tympanoplasty, with a partial ossicular replacement prosthesis. Twenty-four hours later, she developed sudden-onset severe vertigo on bending, and presented 4 days later with nystagmus, dysgeusia on the left side of her tongue, and left-sided tinnitus with profound sensorineural hearing loss (SNHL). Blood serum analysis, including an autoimmune screen was unremarkable. Apart from the SNHL, her symptoms improved with physiotherapy, steroids and antibiotics. A radiological diagnosis of tympanogenic labyrinthitis or haemorrhage was made. Three months later, she developed left-sided headache, associated with blurred vision in the left eye and epiphora, followed by a House-Brackman grade III facial nerve palsy 4 days later. She was started on oral ciprofloxacin and steroids. The facial nerve palsy resolved completely after four weeks.

\section{Radiological findings}

Initial imaging showed no evidence of prosthetic or ossicular chain displacement. Dr Siddiqui demonstrated loss of fluid from the inner-ear structures, which enhanced with contrast, this being consistent with labyrinthitis. Repeated computed tomography at the time of the facial palsy showed osteolysis around the cochlea and the labyrinthine portion of the facial nerve. Dr Siddiqui explained that this is consistent with inflammation.

\section{Conclusion}

Taking into account the post-operative clinical presentation and radiological imaging, we concluded that the patient had developed a rare case of progressive aggressive labyrinthitis. 